pounds are kinetically inert (the corrinoids are not); that a compound containing a metal-alkyl bond is extremely unstable (the methyl-corrinoids are quite remarkably stable towards acid, alkali, and heat, although they are photosensitive); that cobalt(III) complexes are six-coordinate (many of the organo-cobalt(III) complexes are pentacoordinate); and that the properties of metal complexes are influenced by crystal field effects (no crystal field effects are observed with $B_{12}$ derivatives).

Because corrinoids contain stable Coalkyl bonds (they are the only examples of stable naturally occurring organometallics) they have contributed significantly to studies of $\sigma$-bonded organoligands, in contrast to the more commonly studied $\pi$-bonded ligands. Similarly, the photochemistry of metal complexes, initiated by internal transitions on the ligand, has been further developed by recent work on $B_{12}$.

This book would seem to represent required reading for anyone professionally interested in bioinorganic chemistry, but biochemists and coordination chemists generally would gain much from it. It is very readable and the chapters liberally cross-referenced. At $£ 6.00$ it is good value for money.

\section{A. MCAuliffe}

\section{Evolution of Nutrition}

Biology of Nutrition: The Evalution and Nature of Living Systems: The Organization and Nutritional Methods of Life Forms. Edited by Richard N. T. W. Fiennes. Pp. xv+681. (Pergamon: Oxford, February 1972.) £18.

ThIs book of almost 700 pages endeavours to combine a textbook of biochemistry, a textbook of ecology, a treatise on the origin of life (emphasizing that the protocells, borrowing organic molecules from the medium, represent the first forms of nutrition), a treatise on bioenergetics, and a treatise on biochemical evolution, these additional texts being provided as the accessory knowledge necessary for the perusal of the textbook on nutrition in the context of evolution, which the reader is expected to untangle from this skein.

The evolution of nutrition and of chemical needs depends, on the one hand, on the evolution of ATP-generating processes, and on the other hand, on losses of biosynthetic capacities. These aspects of molecular evolution do not appear clearly defined or placed in order in the book as one would wish, and the distribution of the material remains traditional, placed along the taxa of organisms. Perusing the table of contents, the reader will be able to identify data on a number of nutritional sys- tems: bacteria and micro-fungi, viruses, protozoa, fungi, lichens, marine algae, pelagic invertebrates, worms, echinoderms, coelenterata, molluscs, tunicates, crustacea, insecta, fishes, amphibia, reptiles, birds ... indeed, all living creatures with the exception of man.

Little attention is given to the mechanisms of molecular evolution, and to the variations of metabolic pathways along phylogeny, to account for chemical needs. A consideration of the role of ecomones in the establishment of relations between organisms in the networks of the biochemical continuum is also lacking.

In our present concepts, the appearance in history of the different ATP. generating processes may be considered as having taken place in the order (1) fermentation, leading to (2) photoorganotrophy, leading to (3) photolithotrophy, leading to (4) phytotrophy (plant photosynthesis), leading to (5) oxidative phosphorylation (respiration). By using different parts of the book, the reader will be able to collect information on these successive steps or at least on some of them.

The book is divided into five sections among which the monographic treatments of taxonomic groups are more or less clearly placed in order. Section I is devoted to the evolution of the Earth and to prebiotic chemical evolution and in this section are also included a number of chapters on bacteria, viruses and protozoa, defined as "the earliest life forms existing today".

Section II deals with the acquisition of free energy and its relation to nutrition and growth, while Section III is concerned with the evolution of the habitat. In Section IV emphasis is placed on the living communities and ecological and ethological aspects.

The general leading idea of the book seems to be focused on the stages from the trappings of energy to the nutritional systems of the life of higher organisms. Many of these stages still persist on the Earth and they are necessary for the continuation of man's life. The popular appeal of the concept of pollution is thereby introduced into the book.

As stated by the editor, the volume was originally planned to deal with the nutrition of lower organisms, by which he understands all forms of life except man and domestic animals, whose nutrition is already dealt with in another volume of the same series. The patient reader will be able to extract from the mass of ornaments with which it is overburdened an original book on the nutrition of "lower organisms" written by competent experts. Contrary to the fears of the editor, this comparative treatment of nutrition is not "unregarding" nor "dull" nor "prosy", but, on the contrary, very useful.

MARCEL FLORKIN

\section{Scientific Teaching}

Instructional Science : An International Journal. Editorial policy board: G. F. Brieske, B. N. Lewis, M. MacDonaldRoss and R. Wm. Smith. Vol. 1, No. 1. Pp. 151. (Elsevier: Amsterdam, March 1972.) Institutional subscribers, Dfl. $87.50, \$ 27.30, £ 10.60$. Personal subscriptions, Dfl. $45.50, \$ 14.20, £ 5.50$.

Instructional Science is a new journal in the general area of educational technology. Its declared aim is to publish material relating to teaching, training or communication in general, that is, "with the whole range of processes and considerations which can influence, either directly or indirectly, the impact and effectiveness of attempts to instruct". These are said to range from teaching in the nursery or the classroom to the kinds of instruction attempted by politicians, lawyers, churchmen and the mass media.

The editorial board has wisely maintained a practical slant by using the word "instruction" rather than "learning" in the title. They are obviously aware of the necessity to avoid becoming immersed in material bearing on the learning process itself. We are told that "certain kinds of manuscript on the subject of learning-experiments with rats and pigeons, abstract excursions into automata theory, investigations into the biological bases of memory"-may be refused.

The first article by Professor G. P. Meredith consists of a long, personal and interesting review of its author's work and ideas on epistemics. It is fitting that a new journal should permit itself the luxury of publishing this type of material on rare occasions, but it sets a high standard for what is to follow, and the second article, "Perception of the Future and the Future of Perception", might best have been omitted. It also is discursive, but technically inaccurate, and as a medium of instruction difficult to comprehend.

The remaining three articles are (1) "Style and Effectiveness in Education and Training", by Ivor K. Davies; (2) "Reflections on the Process of Visual Design", by Nicholas Tomlinson and John Stevens; and (3) "Computer Frequency Control of Vocabulary in Language Learning Reading Materials", by D. Barton Johnson. These are of a high standard. Book reviews and correspondence are also included.

Instructional Science, besides acting as a general forum for those interested in the technical aspects of instruction, will be of use to those involved with instruction of one form or another, but who have difficulty isolating useful material bearing directly on this topic from the mass of articles published in the journals.

C. E. M. Hansel 